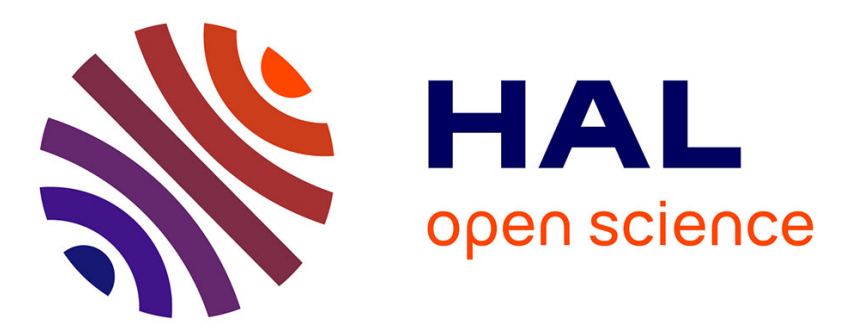

\title{
Anxiété, dépression et partage social des émotions : Des stratégies de régulation émotionnelle interpersonnelle spécifiques
}

\author{
Aurélie Pasquier, Agnès Bonnet, Jean-Louis Pedinielli
}

\section{To cite this version:}

Aurélie Pasquier, Agnès Bonnet, Jean-Louis Pedinielli. Anxiété, dépression et partage social des émotions: Des stratégies de régulation émotionnelle interpersonnelle spécifiques . Journal de Thérapie Comportementale et Cognitive, 2008, 18 (1), pp.2-7. 10.1016/j.jtcc.2008.02.007 · hal-01271909

\section{HAL Id: hal-01271909 \\ https://hal-amu.archives-ouvertes.fr/hal-01271909}

Submitted on 9 Feb 2016

HAL is a multi-disciplinary open access archive for the deposit and dissemination of scientific research documents, whether they are published or not. The documents may come from teaching and research institutions in France or abroad, or from public or private research centers.
L'archive ouverte pluridisciplinaire HAL, est destinée au dépôt et à la diffusion de documents scientifiques de niveau recherche, publiés ou non, émanant des établissements d'enseignement et de recherche français ou étrangers, des laboratoires publics ou privés. 
Anxiété, dépression et partage social des émotions :

Des stratégies de régulation émotionnelle interpersonnelle spécifiques

\title{
Anxiety, depression and social sharing of emotions : Specifics interpersonal emotional regulation strategies
}

\author{
Aurélie Pasquier (1), Agnès Bonnet (2), Jean-Louis Pedinielli (3) \\ 1 Attaché Temporaire d'Enseignement et de Recherche*** \\ 2 Maître de Conférence en Psychologie* \\ 3 Professeur de Psychopathologie*
}

*Centre de recherches en Psychologie de la Connaissance, du Langage et de l'Emotion (PsyCLE, EA3273).

Axe thématique III « Emotions, Construction du réel et Pathologie ».

Université de Provence

21, avenue Robert Schuman

13621 Aix-en-Provence Cedex 1

**Département Adaptation Scolaire et Scolarisation des élèves Handicapés (ASH)

Université de Provence

Institut Universitaire de Formation des Maîtres d'Aix-Marseille I

2, avenue Jules Isaac

13626 Aix-en-Provence Cedex 1

Article publié dans le Journal de Thérapie Comportementale et Cognitive en février 2008 (vol. $18 \mathrm{n}^{\circ} 1, \mathrm{p} .2-7$ ) 


\section{Résumé :}

Les états anxieux et dépressifs représentent respectivement $12 \%$ de la population générale. Ils sont associés à des dimensions d'alexithymie et notamment aux difficultés de verbalisation des émotions (Berthoz et coll., 1999). L'objectif de cette recherche est d'identifier les relations pouvant exister entre l'anxiété, la dépression et le partage social des émotions considéré comme un processus de régulation émotionnelle interpersonnelle (Rimé, 2007). La méthodologie quantitative prend appui sur un échantillon de 107 patients de médecine générale et d'individus tout-venant $(\mathrm{H} / \mathrm{F})$, caractérisés par la présence ou l'absence d'anxiété état et de dépression état. Nous avons évalué les états anxieux et dépressifs (HAD) et les composantes du partage social des émotions (échelle de PSE). Les résultats montrent l'existence de relations positives entre l'inhibition du partage social émotionnel et l'anxiété état, et entre l'inhibition d'une partie seulement du vécu émotionnel lors du partage et le niveau de dépression. Il semble que dans le premier cas il s'agit d'une stratégie visant à éviter la réactivation du vécu émotionnel alors que dans le deuxième cas, l'individu chercherait davantage à se préserver d'une vision négative de soi.

Mots-clés : anxiété, dépression, partage social des émotions, alexithymie.

\section{Summary :}

Depressed and anxiety states represent respectively $12 \%$ of general population. They are associated with alexithymia dimensions like difficulties in verbalization of emotions (Berthoz et coll., 1999). The aim of the research is to identify the relations between anxiety, depression and social sharing of emotions considered as a process of interpersonal emotional regulation (Rimé, 2007). A quantitative method was used. 107 patients of general medicine and control subjects were characterised by the presence or the absence of anxiety and depressed states. We measured depressed and anxiety states with the Hospital Anxiety and Depression Scale (HAD) and le components of social sharing of emotions. Results point out the presence of positives relations between anxiety state and inhibition of social sharing of emotions, and between a part of emotions' inhibition and depression. In anxiety, it's about a strategy used in order to avoid emotions reactivation happening despite of, in depression, the subject would try to protect from a negative perception.

Key words : anxiety, depression, social sharing of emotions, alexithymia. 


\section{Introduction}

L'anxiété et la dépression sont des troubles émotionnels qui touchent respectivement $12 \%$ de la population générale. Cependant, les études épidémiologiques montrent que concrètement les individus qui souffrent de ce type de symptômes n'entament généralement pas de démarche visant à consulter un thérapeute, quel que soit son domaine d'exercice (Agence du médicament, 1998). Deux tiers des individus dépressifs sont pris en charge uniquement par des médecins généralistes, et les trajectoires des individus anxieux semblent similaires (Rouillon et coll., 1993). De plus, les études scientifiques montrent l'existence de relations positives entre les symptomatologies anxieuses et dépressives, et des difficultés dans l'identification et la description des émotions (Honkalampi et coll., 2000). Ces difficultés correspondent à ce qui est plus communément appelé «l'alexithymie ». Ce terme proposé par Sifnéos (1973) signifie littéralement l'absence de mots pour décrire ses émotions. Ce concept a été plus largement défini comme des difficultés à identifier, distinguer et verbaliser ses états émotionnels, ainsi qu'une vie imaginaire réduite et un mode de pensée dit «opératoire », c'est-à-dire dépourvu de tout contenu affectif (Luminet, 2002).

Cependant, même si ces manifestations peuvent se retrouver dans la pratique clinique auprès des patients, il semble qu'une autre raison peut être avancée concernant l'obstacle principal des individus souffrant de symptômes anxieux et/ou dépressifs à parler de leurs difficultés. En effet, le vécu même des maladies anxieuse et dépressive (tension interne, culpabilité, désespoir,...) peut freiner toute demande de soin. La stigmatisation des termes de dépression et d'anxiété participe sans doute aux réticences que peut manifester l'individu à faire état de ses difficultés. La dimension sociale prend alors toute son importance et semble constituer un facteur important dans le refus du sujet au suivi, quel qu'il soit. Plus largement on peut se demander qu'est-ce qui peut empêcher le sujet à verbaliser ses émotions ?

\section{Description et définition des états anxieux et dépressifs}

A l'origine, l'anxiété facilite l'adaptation même si elle est déplaisante : elle peut servir à mobiliser les ressources pour permettre des changements bénéfiques et peut faciliter le développement psychologique (Taylor et coll., 1992). Les limites entre la réaction anxieuse normale qui mobilise l'individu pour affronter une situation difficile et l'anxiété pathologique sont floues. L'anxiété est pathologique quand elle n'est plus liée à des événements de la vie et qu'elle survient sans raison. Nous choisissons ici d'utiliser le terme d' « état anxieux » dans la 
mesure où nous nous intéressons à «l'état émotionnel de tension nerveuse » (Postel, 1998) subjectivement ressenti par l'individu. Cet état est plus ou moins stable et il peut s'accompagner de signes tels que l'agitation motrice et des manifestations physiologiques d'anxiété (boule dans la gorge, ventre noué, etc.).

D’un point de vue clinique, l'anxiété peut être associée à des symptômes dépressifs. En effet, le clinicien retrouve souvent dans sa pratique la co-existence de symptômes anxieux et dépressifs sans que les uns et les autres ne soient totalement présents pour parler de troubles anxieux et dépressifs. Ainsi nous préférons nous rapprocher d'une définition de la dépression au sens d' "état dépressif », considérée comme un état émotionnel généralement transitoire, formé de quatre composantes que sont l'humeur dépressive, le ralentissement psychomoteur et le pessimisme en l'avenir (Pedinielli et coll., 2002).

\section{Le partage social des émotions : régulation émotionnelle interpersonnelle}

Rimé (1991 ; cité dans Rimé, 2005) a proposé l'expression de «partage social des émotions » (PSE) pour rendre compte de la propension des individus à communiquer avec leur environnement suite à des événements émotionnels. Si les premières observations de ce phénomène sont issues de l'étude des traumatismes émotionnels (catastrophes naturelles, guerres, etc.), un certain nombre de travaux a mis en évidence que le partage social des émotions concerne l'ensemble des événements émotionnels. Les données suggèrent qu'il constitue une conséquence typique de l'émotion voire même une facette de l'expérience émotionnelle. Partager socialement ses émotions permettrait de réguler ses éprouvés subjectifs (Rimé, 2007).

Habituellement, ce n'est pas un travail cognitif de mise en sens de l'événement vécu qui est visé. Les bénéfices sont plus d'ordre sociaux et émotionnels. En effet, des travaux montrent que lorsque l'individu partage socialement ses émotions, les phénomènes physiologiques et émotionnels consécutifs à l'expérience émotionnelle sont améliorés. De plus, un rapprochement affectif se produit renforçant les liens sociaux de l'individu. A l'inverse, l'inhibition du partage social des émotions entraînerait des coûts sociaux et émotionnels pour les deux partenaires d'une conversation émotionnelle (Butler, 2004).

Cette recherche s'intéresse spécifiquement au processus de partage social des émotions chez des individus souffrant de symptômes anxieux et dépressifs. L'objectif de cette étude est d'identifier la régulation émotionnelle interpersonnelle spécifique à chaque état 
émotionnel considéré ici. L'inhibition du partage social des émotions en tant que processus de régulation émotionnelle interpersonnelle, serait spécifique aux états anxieux. Alors que l'inhibition d'une partie seulement du vécu émotionnel lors du partage social des émotions serait davantage le fait d'une symptomatologie dépressive.

\section{Méthodologie}

L'échantillon est constitué de 107 patients (H/F) issus de cabinets de médecine générale et de sujets tout-venant (cf. tableau 1). Les premiers ont été préalablement identifiés comme présentant des symptômes anxieux et/ou dépressifs (critères DSM-IV-TR) par le médecin généraliste. L’âge moyen est de 43,21 ( $\pm 12,76)$ avec un sexe ratio $(\mathrm{H} / \mathrm{F})$ de 13/94. Nous avons constitué deux groupes de sujets selon leur niveau d'anxiété, tout en prenant en compte leur score de dépression. Un premier groupe ( $\mathrm{N}=60$ avec un sexe ratio $\mathrm{H} / \mathrm{F}$ de 7/53) est composé des sujets de médecine générale dont le score atteint la note seuil (égale à 8), note à partir de laquelle on considère que le niveau de symptômes est pathologique (groupe anxieux/dépressifs noté $\mathrm{AD}$ ). Au sein de ce même groupe tous les sujets ont aussi un score de dépression dont certains atteignent la même note seuil que celle employée pour le niveau d'anxiété (nombre d'anxieux et dépressifs sur effectif total du groupe $1: 13 / 60$ ). Un deuxième groupe est constitué de sujets tout-venant n'atteignant aucune des notes seuils permettant de statuer sur le critère pathologique des niveaux d'anxiété et de dépression (groupe non anxieux/dépressifs noté NAD).

\section{$\underline{\text { Tableau } 1}$}

Les variables étudiées sont relatives aux informations issues de l'échelle d'évaluation du processus de partage social des émotions (Rimé, 1991). Nous avons choisi d'utiliser cette échelle en demandant aux sujets de se rappeler un événement émotionnel négatif considéré comme le plus marquant qu'ils aient pu vivre. Ils devaient ensuite répondre à différentes questions en se référant à cet événement. Les variables dépendantes sont l'anxiété état et la dépression état. Elles sont évaluées par l'échelle d'auto-évaluation «HAD » (Hospital Anxiety and Depression Scale; Zigmond et coll., 1983). Cette échelle dimensionnelle en 14 items permet d'évaluer le niveau actuel des symptômes anxieux et dépressif en donnant lieu à deux scores spécifiques à chaque état.

Après avoir rempli individuellement les protocoles à leur domicile, il était demandé aux sujets de l'envoyer sous pli anonyme à l'investigateur de la recherche. Les données ont 
fait l'objet d'un traitement statistique à l'aide du logiciel Statview 5.0. Nous avons procédé à des comparaisons de moyennes ( $\mathrm{t}$ de student) dans le but de montrer l'existence de différences entre nos deux groupes de sujets avant de passer à des analyses corrélationnelles univariées ( $\mathrm{r}$ de Bravais-Pearson) qui nous permettrons de déterminer la présence de relations spécifiques entre états anxieux, états dépressifs et processus de partage social des émotions.

\section{Résultats}

Les sujets anxieux et/ou dépressifs (AD) ont en moyenne un niveau d'anxiété de 12,28 comparativement aux sujets non anxio-dépressifs (NAD) qui obtiennent en moyenne un score de 5,51 à la sous-dimension d'anxiété. La différence entre les deux moyennes est significative ( $\mathrm{t}$ de student significatif à $\mathrm{p}<.0001)$. Le score moyen des sujets $\mathrm{AD}$ atteint la note seuil (note $=8$ ) et permet de les considérer comme anxieux.

Le niveau de dépression des sujets AD est en moyenne de 4,90 alors que celui des sujets NAD est de 2,64 en moyenne. La différence entre les deux groupes est significative (t de student significatif à $\mathrm{p}<.0001$ ) même si aucun des deux groupes de sujets n'atteint la note seuil (égale à 8) permettant de considérer le niveau pathologique des symptômes.

Par ailleurs, quelque soit les symptômes considérés, le groupe AD est homogène quant à l'âge et le sexe. Il n'existe pas d'effet principal du sexe et de l'âge sur le niveau d'anxiété au sein du groupe $\operatorname{AD}\left(\mathrm{F}_{\text {sexe }}(0,68)=0,04 ; \mathrm{p}>.05 ; \mathrm{Fâge}(1,81)=0,12 ; \mathrm{p}>.05\right)$. Il n'existe pas non plus d'effet de l'âge et du sexe sur le niveau de dépression pour le groupe $\operatorname{AD}\left(F_{\text {sexe }}(12,18)=1,48\right.$; $\mathrm{p}>.05$ et $\mathrm{Fâge}(1,28)=0,15 ; \mathrm{p}>.05)$.

\section{Etats anxieux, états dépressifs et partage social des émotions}

Des analyses de corrélations de type Bravais-Pearson entre les états anxieux et dépressifs au sein du groupe AD montrent que le niveau d'anxiété est corrélé positivement de manière significative avec le niveau de dépression $(\mathrm{r}=.34 ; \mathrm{p}=.007)$. Par conséquent, plus les individus présentent une anxiété état et plus ils manifestent un niveau de symptômes dépressifs important.

Nous nous sommes ensuite intéressés aux différences de moyennes entre les deux groupes concernant l'inhibition du partage social des émotions («s'empêcher de parler de l'événement émotionnel avec autrui »). Il n'existe pas de différences significatives entre les deux groupes AD et NAD concernant cette variable ( $t$ de student non significatif). 
Cependant, au niveau qualitatif, il était demandé aux sujets ayant inhibé le partage social de leurs émotions de donner les raisons de leur inhibition. Deux types de réponse ont été formulés, ce qui a donné lieu à la classification suivante :

- absence de partage social pour cause personnelle : «c'est trop douloureux d'en parler », «trop de souffrance ».

- absence de partage social pour cause sociale : «cela ne regarde que moi », «c'est trop intime ».

Les résultats montrent qu'il existe des différences significatives entre les deux groupes concernant l'absence de partage social pour cause personnelle. Les sujets AD ont majoritairement répondu qu'ils inhibaient le partage social de leurs émotions dans le but de ne pas se remémorer l'événement émotionnel négatif (chi-2=3,85; $\mathrm{p}=.05$ ).

Concernant cette fois l'inhibition de certains aspects du vécu émotionnel associé à l'événement émotionnel cible (« expression complète du vécu émotionnel »), il n'existe pas non plus de différences significatives entre les groupes $\mathrm{AD}$ et NAD à cette variable $(\mathrm{t}$ de student non significatif). Cependant, en s'intéressant au contenu, les raisons énoncées par les sujets ayant inhibés une partie du vécu émotionnel sont de deux types :

- raisons personnelles d'inhibition d'une partie du vécu : «C'est trop douloureux, trop difficile de parler de tout. »;

- raisons sociales d'inhibition d'une partie du vécu: «C'est trop intime, trop personnel. », «J'aurais une mauvaise opinion de moi-même si j'en parle aux autres. ». Lorsque certains aspects du vécu émotionnel ont été inhibés lors du partage social des émotions, les sujets $\mathrm{AD}$ rapportent davantage de raisons d'inhibition sociales (chi-2=6,11 ; $\mathrm{p}=.01)$ par rapport aux sujets NAD.

Les analyses de corrélations univariées mettent en évidence l'existence de relations positives significatives au sein du groupe $\mathrm{AD}$, entre les niveaux d'anxiété et de dépression, et les composantes d'inhibition de partage social des émotions :

- plus le niveau d'anxiété est élevé et plus les sujets $\mathrm{AD}$ ont tendance à inhiber le partage social de leurs émotions $(\mathrm{r}=.26 ; \mathrm{p}=.05)$;

- plus le niveau de dépression est élevé et plus les sujets AD inhibent le partage social de leurs émotions $(\mathrm{r}=.27 ; \mathrm{p}=.04)$;

- plus le niveau de dépression est élevé et plus les sujets $\mathrm{AD}$ ont tendance à inhiber une partie seulement de leur vécu émotionnel lors du partage social de leurs émotions $(\mathrm{r}=.33)$. 


\section{Interprétation des résultats}

Les résultats montrent que si les niveaux d'anxiété et de dépression sont positivement corrélés entre eux conformément à la littérature, ils ne présentent pas les mêmes types de relations avec les composantes de partage social des émotions.

L'inhibition du partage social des émotions associées à un événement émotionnel cible est en lien avec le niveau d'anxiété actuel des individus de cette étude. En effet, plus les individus ont eu tendance à s'empêcher de partager socialement leurs émotions à l'issu d'un événement émotionnel et plus ils présentent un niveau d'anxiété élevé.

Le niveau de dépression est lui aussi positivement corrélé à l'inhibition du partage social des émotions, mais il entretient plus spécifiquement une relation positive avec l'inhibition de certains aspects du vécu émotionnel lors du partage social des émotions. On peut penser que la relation qui existe entre dépression et inhibition du partage social des émotions est en grande partie le fait du niveau d'anxiété élevé présenté chez les mêmes sujets.

Si les individus anxieux inhibent le partage social de leurs émotions, les individus dépressifs ont quant à eux tendance à partager leurs émotions mais en taisant une partie de leur vécu émotionnel.

Par ailleurs, si on s'intéresse au contenu des réponses des individus $\mathrm{AD}$, on remarque que lorsqu'ils inhibent le partage de leurs émotions, il s'agit de raisons qualifiées de personnelles du type «c'est trop douloureux, c'est trop de souffrance ». En effet, ces réponses diffèrent de celles qualifiées de «sociales » du type «c'est trop intime, cela ne regarde que moi ». Ainsi les individus qui inhibent le partage de leurs émotions le feraient dans le but de se préserver du rappel d'un événement trop douloureux pour eux. Nous pouvons émettre l'hypothèse selon laquelle l'inhibition du partage social des émotions chez les individus anxieux aurait pour fonction d'éviter la réactivation du vécu émotionnel négatif et intense rattaché à l'événement émotionnel.

Selon Rimé (2007), les individus ont habituellement tendance à partager leurs émotions avec autrui dans le but de recevoir un soutien socio-affectif (réconfort, manifestations d'empathie, etc.) source de bénéfices émotionnels et sociaux. L'inhibition du partage social des émotions permettrait ni de réguler ses émotions ni de recevoir le soutien positif d'autrui. 
L'inhibition de certains aspects du vécu émotionnel lors du partage social des émotions semble spécifique aux individus dépressifs. On peut émettre l'hypothèse que chez les individus dépressifs, ce processus d'inhibition aurait pour fonction de préserver l'individu d'une image négative de soi auprès d'autrui.

Cependant, si les raisons de l'inhibition du vécu émotionnel diffèrent entre les individus anxieux et dépressifs, les conséquences sont sans doute les mêmes : ils ne retirent pas les bénéfices habituellement rencontrés tant au niveau émotionnel que social.

\section{Conclusion}

Les difficultés de verbalisation souvent rencontrées dans les états anxieux et dépressifs (Berthoz et coll., 1999) pourraient dépendre de déterminants subjectifs et sociaux jusqu'ici ignorés. En effet, si il a été montré que l'alexithymie pouvait être envisagée comme une stratégie adaptative (Pedinielli, 1992) ou encore comme un facteur modérateur du fonctionnement émotionnel (Bréjard et coll., 2005 ; Pasquier et coll., 2007), au-delà des conceptions en termes de trait de personnalité, il n'en est pas moins difficile d'appréhender les déterminants de cette stratégie ainsi que ses conséquences.

Les individus anxieux inhiberaient le partage de leurs émotions avec autrui dans le but de ne pas réactiver l'événement émotionnel source d'un vécu émotionnel négatif. La contrepartie de cette stratégie adaptative est non sans conséquence pour l'individu qui se prive d'un moyen de régulation émotionnelle efficace. On peut penser que par cette stratégie, les individus se préservent d'une situation bien plus difficile pour eux, celle de donner un sens à leur vécu.

Nous avons montré précédemment (Pasquier, Bonnet et Pedinielli, 2007) le rôle de l'intensité émotionnelle dans l'anxiété. L'intensité des émotions, ressentie subjectivement par l'individu, rendrait difficile le travail cognitif. Les individus anxieux manifesteraient un manque de clarté cognitive venant renforcer leur état émotionnel. Inhiber ses émotions négatives permettrait aux individus de ne pas se confronter à ce qui les rend anxieux (rappel de l'événement émotionnel) et à ce qui renforce leur anxiété (chercher à donner du sens à leur vécu subjectif).

Concernant la symptomatologie dépressive, il semble qu'elle soit davantage en lien avec l'inhibition d'une partie du vécu émotionnel, ceci dans le but de se préserver d'une image négative de soi auprès d'autrui. Conformément à la littérature nous pouvons penser que ce 
processus d'inhibition entraîne des coûts émotionnels et sociaux pour l'individu (Butler, 2004 ; Gross, 2002). Cette stratégie ne permet pas à l'individu de réguler de manière efficace ses émotions et instaure de surcroît une distanciation des liens sociaux. Si l'individu cherche à se protéger d'une image négative de soi, cette protection escomptée se fait au détriment d'une consolidation des liens à autrui.

On peut envisager la spécificité de chaque type de régulation en fonction du vécu même de chaque état émotionnel considéré ici.

Le tableau anxieux se compose de manifestations telles qu'une tension interne forte et mal différenciée. Ici l'intensité émotionnelle domine et l'individu chercherait à faire diminuer cette tension interne par l'inhibition du partage social de ses émotions. Le tableau dépressif se compose quant à lui de manifestations telles qu'une intense tristesse et une perte des éprouvés de plaisir.

Cependant, si on connaît les déterminants internes des états dépressifs (Pedinielli et coll., 2002), cette étude nous permet d'aborder l'influence du regard d'autrui dans la verbalisation des émotions. En effet, nous venons de montrer que si les individus dépressifs parlent de leurs émotions c'est souvent de manière incomplète. Une partie du vécu émotionnel ne peut être soumise au regard des autres.

Notre étude questionne le concept d'alexithymie jusqu'ici abordé à travers des déterminants internes aux individus. En ce sens, nous rejoignons une étude qui rapproche le concept d'alexithymie de celui de la «honte sociale» (Suslow et coll., 2000). Si l'individu exprime peu ou pas d'émotions, ce n'est sans doute pas seulement pour des raisons de difficultés d'identification mais peut être bien plus pour des raisons sociales. Il s'agirait davantage de ce que l'individu choisit ou non de dire à autrui.

\section{Bibliographie}

Agence du médicament. Etude de la prescription et de la consommation des antidépresseurs en ambulatoire, Observatoire national des prescriptions et consommations des médicaments 1998.

Barczack, P., Kane, N., Andrews, S., Congdon, A.M., Clay, J.C., \& Betts, T. Patterns of psychiatric morbidity in a genito-urinary clinic: a validation of the Hospital Anxiety Depression scale (HAD). Brit. J. Psychiatry 1988 ; 152 : 698-700. 
Berthoz, S., Consoli, S., Perez-Diaz, F., \& Jouvent, R. Alexithymia and anxiety : compounded relationships ? A psychometric study. European Psychiatry 1999 ; 14 : 372-378.

Bréjard, V., Bonnet, A. et Pedinielli, J-L. Régulation des émotions, dépression et conduites à risques : l'alexithymie comme facteur modérateur, Annales Médico-Psychologiques $2005 ; 613: 1-9$.

Butler, E.A. Emotional conversations: regulation of emotions. Dissertation Abstracts International Section B. The Sciences \& Engineering 2004 ; 65(4-B), 2082-94.

Gross, J.J. Emotion regulation: Affective, cognitive, and social consequences. Psychophysiology $2002 ; 39,281-91$.

Honkalampi K., Hintikka, J., Tanskanen, A., Lehtonen, J., Viinamaki, H. Depression is strongly associated with alexithymia in the general population. Journal of Psychosomatic Research 2000 ; 48 : 99-104.

Luminet, O. (2002). Psychologie des émotions: confrontation et évitement. Bruxelles : De Boeck Universités.

Pasquier, A., Bonnet, A., \& Pedinielli, J.-L. Fonctionnement cognitivo-émotionnel : le rôle de l'intensité émotionnelle chez les individus anxieux. Annales Médico-psychologiques, sous presse.

Pedinielli, J.L. Psychosomatique et alexithymie. Paris : PUF. 1992.

Pedinielli, J.L. \& Bertagne, P. Les névroses. Paris : Nathan. 2002.

Postel, J. Dictionnaire de psychiatrie et de psychopathologie clinique. Montréal : Larousse Bordas. 1998.

Rimé, B. Le partage social des émotions. Paris : Presses Universitaires de France. 2005.

Rimé, B. Interpersonal emotion regulation. In J.J. Gross (Ed.), Handbook of emotion regulation (pp. 466-485). New York: The Guilford Press. 2007.

Rouillon, F., Lejoyeux, M., \& Le Gep, F. Modalités d'accès aux soins en consultation psychiatrique, Ann. Méd. Psychol. 1993 ; 151(9) : 628-632.

Sifnéos, PE. The prevalence of alexithymic characteristics in psychosomatic patients. Psychother Psychosom 1973 ; 22 : 255-62.

Suslow, T., Donges, U.-S., Kersting, A. \& Arolt, V. (2000). 20-Item Toronto Alexithymia Scale: Do difficulties describing feelings assess proneness to shame instead of difficulties symbolizing emotions? Scandinavian Journal of Psychology, 41(3), 329334.

Taylor, L., Gorman, J. Theoritical and therapeutic considerations for anxiety disorders. Psych Quaterly $1992 ; 63(4): 319-42$. 
Zigmond, A.S., \& Snaith, R.P. The Hospital Anxiety and Depression Scale. Acta Psychiatr. Scand 1983, $67: 361-370$. 


\begin{tabular}{l|l|l|l} 
& Sujets MG & Sujets PG & Total \\
\hline Etats anxieux & 47 & 0 & 47 \\
\hline Etats anxieux et dépressifs & 13 & 0 & 13 \\
\hline $\begin{array}{l}\text { Absence d'anxiété/dépression } \\
\text { pathologique(s) }\end{array}$ & 0 & 47 & 47 \\
\hline Total & $\mathbf{6 0}$ & $\mathbf{4 7}$ & $\mathbf{1 0 7}$ \\
\hline
\end{tabular}

Tableau 1: Répartition des sujets en fonction de l'état émotionnel et leur provenance 